\title{
Effect of Alkali Compound on Morphology and Luminescence Intensity of Europium-doped Yttria
}

\author{
Astuti, Mikrajuddin Abdullah ${ }^{(a)}$, and Khairurrijal \\ Physics of Electronic Materials Research Division, \\ Faculty of Mathematics and Natural Sciences, \\ Institut Teknologi Bandung \\ ${ }^{(a)}$ Correspondence author: e-mail:din@fi.itb.ac.id
}

\begin{abstract}
Luminescent particles of europium-doped yttria $\left(\mathrm{Y}_{2} \mathrm{O}_{3}: \mathrm{Eu}\right)$ have been synthesized by heating nitrous precursors in a solution of polyethylene glycol (PEG) matrix containing alkali compound ( $\mathrm{LiOH}, \mathrm{NaCl}, \mathrm{KCl}$, and $\mathrm{CaCl}_{2}$ ). $\mathrm{High}$ luminescence intensity at around $612 \mathrm{~nm}$ contributed by electron transitions in $E u^{3+}{ }^{5} D_{0} \rightarrow{ }^{7} F_{0},{ }^{5} D_{0} \rightarrow{ }^{7} F_{1},{ }^{5} D_{0} \rightarrow$ ${ }^{7} F_{2}$, and ${ }^{5} D_{0} \rightarrow{ }^{7} F_{3}$ ) were observed. The highest intensity was observed in samples prepared using Li compound, but the smallest particle size was observed in samples prepared using Ca compound. This approach is promising for production of submicrometer down to nanometer-sized $\mathrm{Y}_{2} \mathrm{O}_{3}$ :Eu particles that have both small in particle sizes and high luminescence intensity which is potential for use in development of luminescence ink that can be adapted to the current ink jet printing technology.
\end{abstract}

Keywords: Luminescence spectra, Polyethylene glycol, Europium doped-yttria $\left(\mathrm{Y}_{2} \mathrm{O}_{3}: \mathrm{Eu}\right)$

\section{Introduction}

We have reported previously the synthesis of europium-doped yttria $\left(\mathrm{Y}_{2} \mathrm{O}_{3}: \mathrm{Eu}\right)$ particles by heating the nitrous metals in a solution of polyethylene glycol. The role of polymer is to reduce the size of particles. We have reported the production of various oxide particles using this method ${ }^{1-12)}$. Some of the particles, especially luminescent particles, have been used to develop luminescent ink for various security applications $^{10,11)}$. However, we still face some problems with these particles, such as the particle sizes are still larger (mostly in submicrometer) even the crystalline size could be less than $100 \mathrm{~nm}$. This large in size caused the problem of making luminescent ink using the present ink jet printer to developed security codes.

Reducing in the particle sizes is strictly important for development of compatible ink for the present ink jet printing technology. In this work, we try to reduce further the size of particles by adding relatively large amount of alkali compounds in the polymer solution. The large amount of alkali compound might prevent agglomeration of bornnucleis. Therefore each nuclei will be surrounded by alkali compound to reduce the tendency of making contacts with another nuclei. By heating, each particle is still separated from the others so that the uncontrolled size growth can be avoided. After heating, the material is then washed with water several times (by using ultrasonic bath) to remove the alkali compound and resulting alkali compound-free $\mathrm{Y}_{2} \mathrm{O}_{3}$ :Eu particles.

In addition, adding alkali in the precursor might increase the photoluminescence intensity. There are some reports on the enhancement of the luminescence intensity in a lithium-doped $\mathrm{Y}_{2} \mathrm{O}_{3}$ : Eu particles ${ }^{13-15)}$. Therefore, using the present method we expect to achieve two goals, i.e., to reduce the particle sizes (due to avoiding agglomeration of born nuclei by compound) and to improve the luminescence intensity.

\section{Experiment}

Similar to that have been reported by Astuti et $a l^{10,11)}, \mathrm{Eu}\left(\mathrm{NO}_{3}\right)_{3} \cdot 6 \mathrm{H}_{2} \mathrm{O}$ (Kanto Chemicals, Japan) 0.1 $\mathrm{g}$ and $\mathrm{Y}\left(\mathrm{NO}_{3}\right)_{3} \cdot 6 \mathrm{H}_{2} \mathrm{O}$ (Kanto Chemicals, Japan) $2 \mathrm{~g}$ were dissolved in $100 \mathrm{~mL}$ of a distiled water. The molar fraction of Eu/Y was fixed at $4 \% \mathrm{~mol} / \mathrm{mol}$. This is the the optimum doping level of europium in a yttrium oxide host reported previously to obtain the highest photoluminescence (PL) intensity ${ }^{10,11)}$. After completely disolving these of two nitrous metals, $8 \mathrm{~g}$ of polyethylene glycol (PEG, Wako pure chemical, Japan) was added into the solution and then stirred with heating at $100{ }^{\circ} \mathrm{C}$.

Alkali compounds $\left(\mathrm{NaCl}, \mathrm{KCl}\right.$, and $\mathrm{CaCl}_{2}$ or $\mathrm{LiOH} .2 \mathrm{H}_{2} \mathrm{O}$, Bratachem, Indonesia) of $0.5 \mathrm{~g}$ was dissolved in $10 \mathrm{~mL}$ of a distiled water. Two mixtures were then mixed for 2 hours at $100{ }^{\circ} \mathrm{C}$. Then the mixture was then heated in a furnace at $1000{ }^{\circ} \mathrm{C}$ for one hour. Another mixture of Y/Eu/PEG (without alkali compound) was also made for a comparison. The scheme of sample preparation is illustrated in Fig. 1. A fraction of samples made with alkali compounds were then washed with distilled water in an ultrasonic bath to remove alkali compound. Finally the samples were then dried and characterized.

The morphology of samples were recorded using a field emission scanning electron microscopy (FE-SEM Hitachi S-5000) instrument. The X-ray diffraction spectra were recorded by a P1710 Philips Analytical instrument. The photoluminescence spectra were recorded using a Shimadzu RF-5301 PC instrument. 


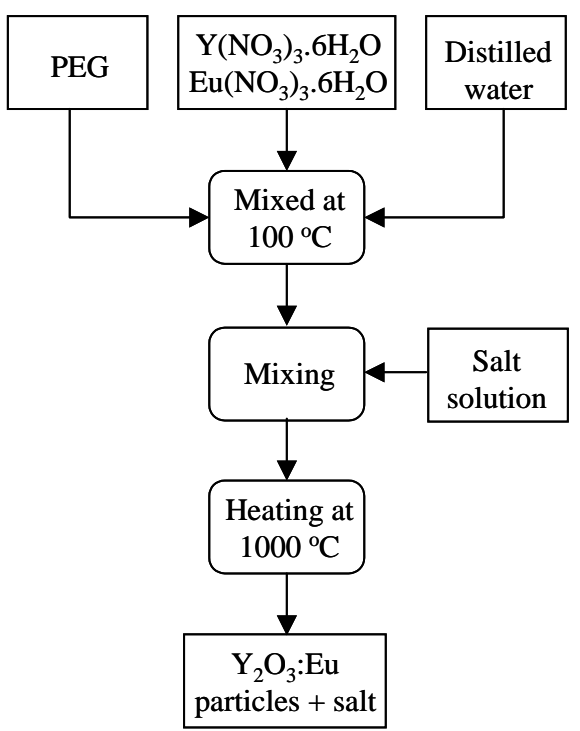

Figure 1 The scheme of samples preparation

\section{Results and Discussion}

\subsection{Photoluminescence}

Figures 2 (a), (b), (c), (d), (e), show the photoluminescence spectra of Li-doped, Ca-doped, Kdoped, Na-doped $\mathrm{Y}_{2} \mathrm{O}_{3}: \mathrm{Eu}$ and a pure $\mathrm{Y}_{2} \mathrm{O}_{3}: \mathrm{Eu}$ detected at room temperature. All samples were prepared at the same conditions. The spectra was dominated by the red emission peak at $612 \mathrm{~nm}$, resulted by ${ }^{5} \mathrm{D}_{0} \rightarrow{ }^{7} \mathrm{~F}_{2}$ transition in $\mathrm{Eu}^{3+}$ ions. The Lidoped $\mathrm{Y}_{2} \mathrm{O}_{3}: \mathrm{Eu}$ sample shows the highest photoluminescence intensity compared to other samples. The luminescence results show that the photoluminescence intensity of Li-doped $\mathrm{Y}_{2} \mathrm{O}_{3}$ :Eu is about 2 times higher than that of alkali-free $\mathrm{Y}_{2} \mathrm{O}_{3}$ :Eu. The improvement in the photoluminescence intensity in the Li-doped samples may result not only from improved crystallinity that leading to higher oscillating strengths for the optical transitions, but also to reduced internal reflections caused by rougher surfaces. The increase in the $\mathrm{Y}_{2} \mathrm{O}_{3}$ :Eu crystallinity by $\mathrm{Li}$ doping would also be expected to yield the improvement of PL brightness.

\subsection{X-ray diffraction}

The increase in $\mathrm{Y}_{2} \mathrm{O}_{3}$ :Eu crystallinity by $\mathrm{Li}$ doping is clear from the XRD patterns as shown in Fig. 3. The crystallinity of samples containing $\mathrm{Li}$ is the highest, the factor that responsible for enhancing the luminescence intensity. Many authors have reported the $\mathrm{Y}_{2} \mathrm{O}_{3}$ :Eu having better crystallinity emit intense luminescence. Improvement of the crystallinity can be obtained by preparing samples at higher temperatures or sintering the samples at longer times.

The XRD patterns of all samples show the occurrence of yttria peaks. The compounds did not change the crystal structure of the samples, but only affect the crystallinity.

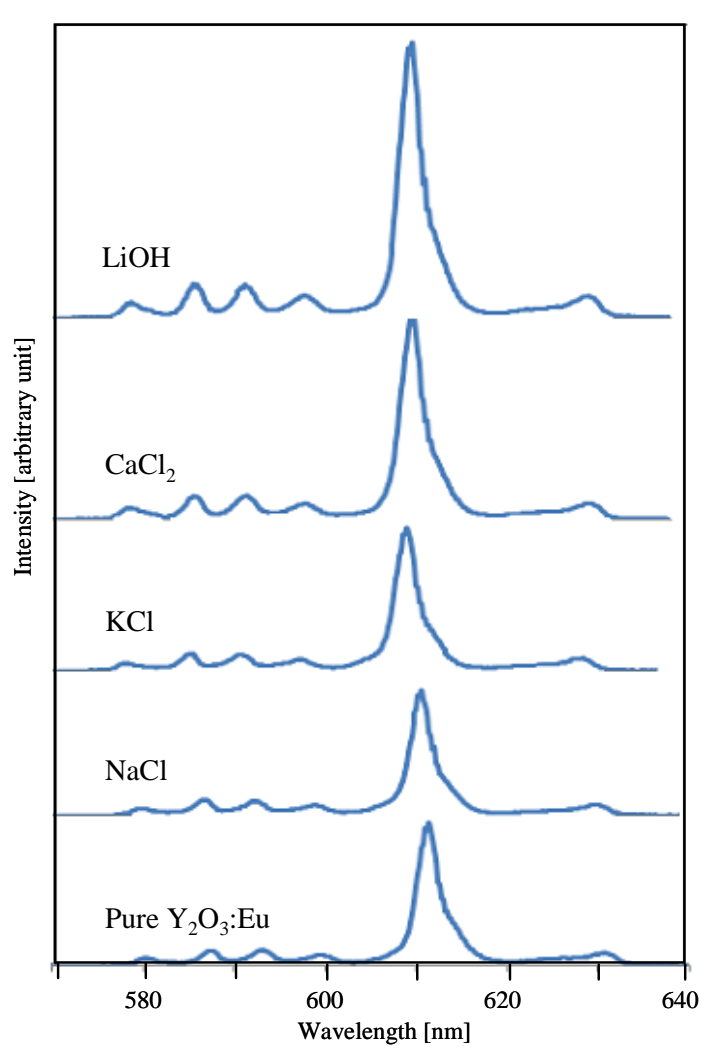

Figure 2. Photoluminescence spectra of $\mathrm{Y}_{2} \mathrm{O}_{3}$ :Eu samples prepared using different alkali: (a) $\mathrm{LiOH}$, (b) CaCl2, (c) $\mathrm{KCl}$, (d) $\mathrm{NaCl}$, and (e) $\mathrm{Y}_{2} \mathrm{O}_{3}$ :Eu without addition of any compounds.

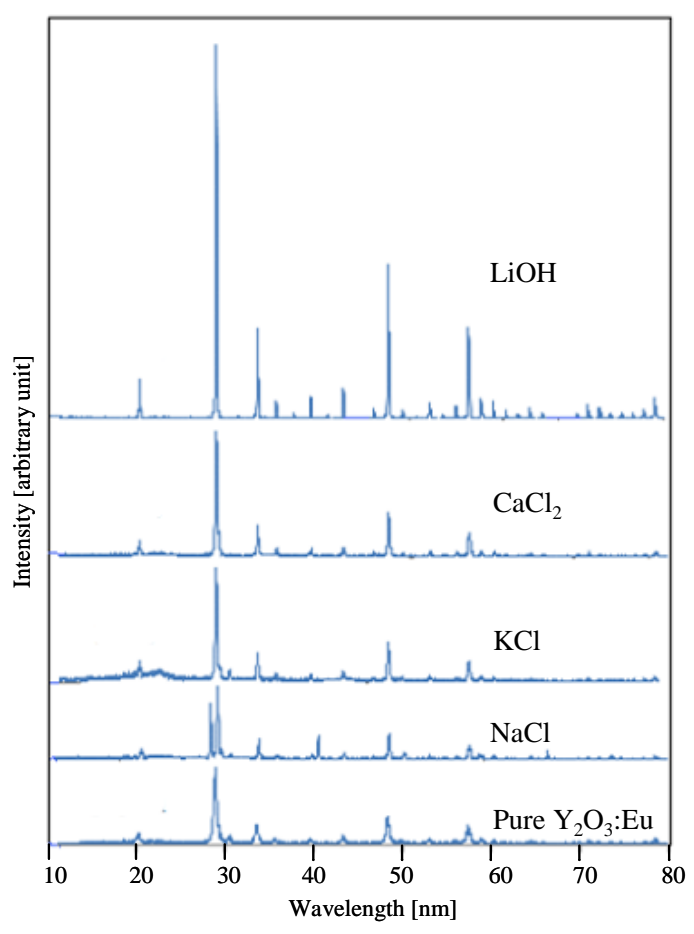

Figure 3. XRD patterns of $\mathrm{Y}_{2} \mathrm{O}_{3}$ :Eu samples prepared using different alkali: (a) $\mathrm{LiOH}$, (b) $\mathrm{CaCl}$, (c) $\mathrm{KCl}$, (d) $\mathrm{NaCl}$, and (e) $\mathrm{Y}_{2} \mathrm{O}_{3}$ :Eu without addition of any alkali compounds. 


\subsection{Morphology}

Figure 4 shows SEM pictures of sample prepared with alkali compound before (left) and after washing from alkali compound (right). We can clearly distinguish the morphology of samples before washing (contain large amount of alkali compound) and after washing (the alkali compounds have been removed). Smaller grain size were obtained in the washed samples because the alkali compounds have been dissolved during washing and removed by filtering.

Fig 4(a) shows that sample prepared using $\mathrm{NaCl}$ still have large granular, which is similar to sample sample prepared with $\mathrm{KCl}$ (Fig. 4(b)). This size is still not suitable for use in developing luminescence ink.
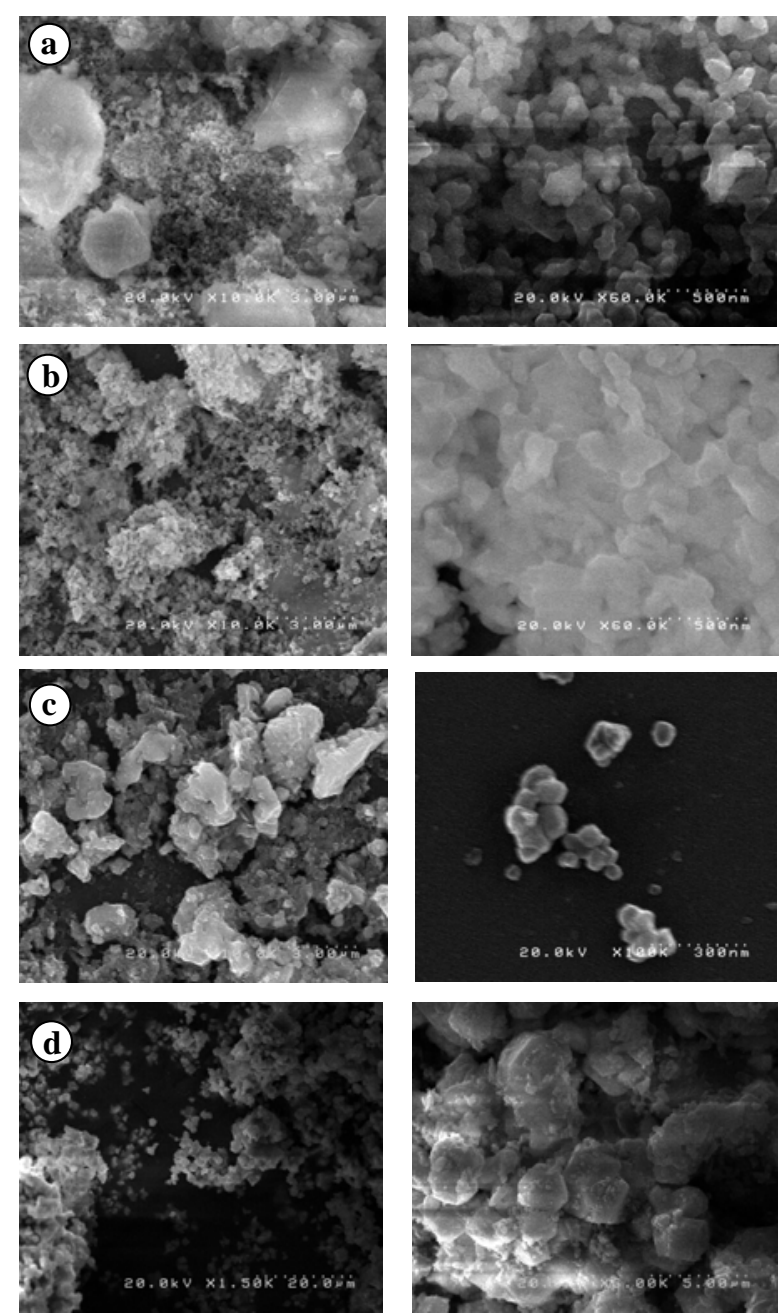

Figure 4. SEM images of samples prepared with addition (a) $\mathrm{NaCl}$, (b) $\mathrm{KCl}$, (c) $\mathrm{CaCl}_{2}$, and (d) $\mathrm{LiOH}$. Left sides are images of samples before removing of the compound and right sides are images of samples after removing $f$ the compound.

The better (smaller) size after washing was obtained in samples prepared by addition of $\mathrm{CaCl}_{2}$ compound, as shown in Fig. 4(c). The particle sizes are less that $100 \mathrm{~nm}$, some are less than $50 \mathrm{~nm}$, and likely separated each other.

Sample prepared using $\mathrm{LiOH}$ is shown in Fig. 4(d). The sizes of granular after washing are very larger, mostly are larger that $5 \mu \mathrm{m}$. This size is larger that the granular size of samples prepared without addition of any alkali compounds, as shown in Fig. 5.

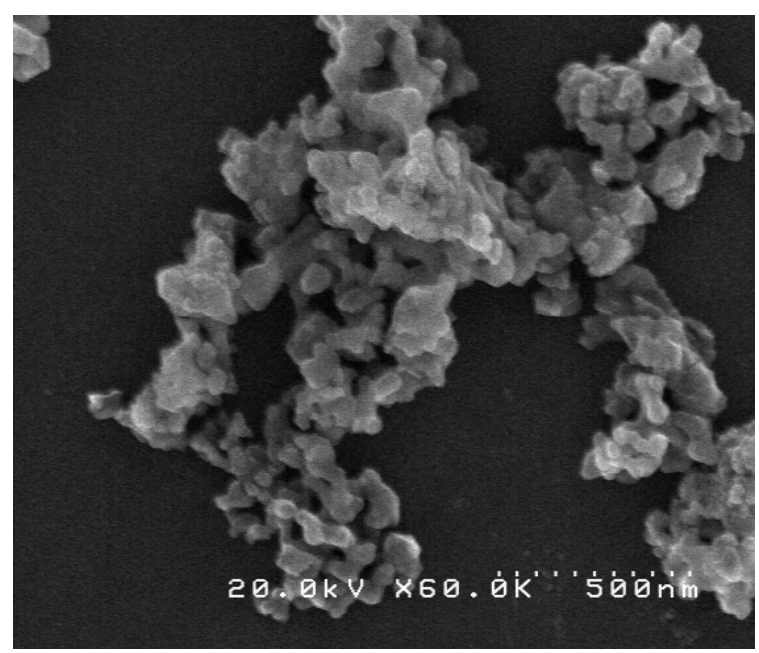

Figure 5. SEM images of samples prepared without addition of any alkali compound.

\section{Conclusion}

Samples of $\mathrm{Y}_{2} \mathrm{O}_{3}: \mathrm{Eu}$ than emit red luminescence have been produced in a solution of polyethylene glycol with addition of large amount of different alkali. We observed that the better luminescence intensity was obtained in sample prepared by the addition of LiOH. This intensity is about twice of that of sample prepared at the same condition without addition of any alkali. This sample also shown the best crystalinity (indicated by the highest XRD peaks). This suggests that the crystalinity is responsible for the enhancement of luminescence intensity. However, sample prepared by addition of $\mathrm{CaCl}_{2}$ compound shows the smallest granular size and also the second in the luminescent intensity. The grain sizes in this sample might be less than $50 \mathrm{~nm}$.

\section{Acknowledgement}

The authors thank Mr. Adi Bagus Suryamas from Hiroshima University for helping some measurements.

\section{References}

1. Liherlinah et al., J. Nano Saintek. 1, 22, 2008.

2. M. Abdullah et al., J. Nano Saintek. 1, 1, 2008.

3. M. Abdullah et al., Powder Technol. 183, 297303, 2008.

4. I. Sriyanti et al., 2007 Conference on Solid State Ionics (Serpong, 1-3 August 2007). 
5. I. Nurhasanah, M. Abdullah, and Khairurrijal, AIP Conf. 989, 147-150, 2008.

6. T. Ogi et al., J. Cryst. Growth 281, 234, 2005.

7. M. Abdullah et al., J. Non-Crystalline Solids 351, pp. 697-704, 2005.

8. M. Abdullah et al., J. Ceram. Soc. Jpn. 113, 97100, 2005.

9. M. Abdullah and K. Okuyama, Proc. ITB Eng. Sci. 36B, 140-152, 2004.

10. Astuti, M. Abdullah, and Khairurrijal, Adv. Optoelectron. Vol 2009, ID 918351, 2009.
11. Astuti, M. Abdullah, and Khairurrijal, J. Nano Saintek. Proc. Sem. Nas. Sains Tekno. Nano, Bandung, June 3, 2009, 61-66, 2009.

12. M. Abdullah et al., Asian J. Energy Environ. (in press, 2009).

13. L. Sun et al., Solid State Commun. 119, 393, 2001.

14. J. H. Jeong et al., J. Phys.: Condens. Matter 15, 567-574, 2003.

15. K.M. Nissamudeen et al., J. Phys. Chem. Solids 70, 821-826, 2009. 